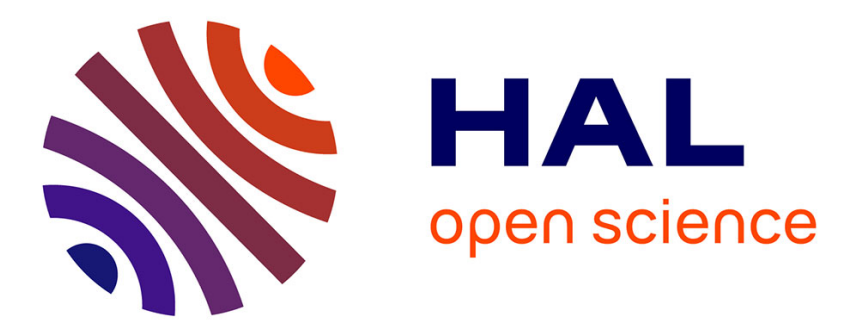

\title{
Strong geometric-phase effects in the hydrogen-exchange reaction at high collision energies: II. Quasiclassical trajectory analysis.
}

Foudhil Bouakline, Suart Althorpe, Pascal Larregaray, Laurent Bonnet

\section{- To cite this version:}

Foudhil Bouakline, Suart Althorpe, Pascal Larregaray, Laurent Bonnet. Strong geometric-phase effects in the hydrogen-exchange reaction at high collision energies: II. Quasiclassical trajectory analysis.. Molecular Physics, 2010, 108 (07-09), pp.969-980. 10.1080/00268971003610218 . hal-00596292

\section{HAL Id: hal-00596292 \\ https://hal.science/hal-00596292}

Submitted on 27 May 2011

HAL is a multi-disciplinary open access archive for the deposit and dissemination of scientific research documents, whether they are published or not. The documents may come from teaching and research institutions in France or abroad, or from public or private research centers.
L'archive ouverte pluridisciplinaire HAL, est destinée au dépôt et à la diffusion de documents scientifiques de niveau recherche, publiés ou non, émanant des établissements d'enseignement et de recherche français ou étrangers, des laboratoires publics ou privés. 


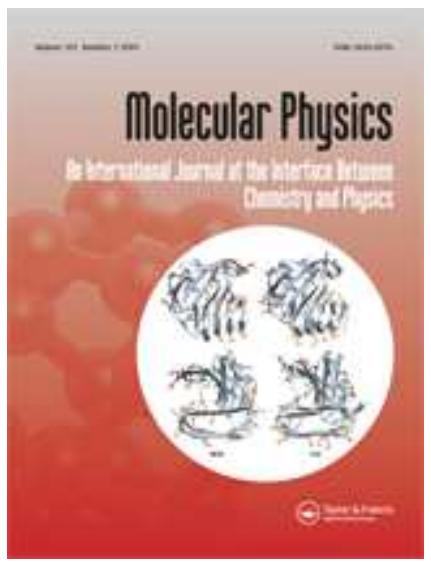

\section{Strong geometric-phase effects in the hydrogen-exchange reaction at high collision energies: II. Quasiclassical trajectory analysis.}

\begin{tabular}{|r|l|}
\hline Journal: & Molecular Physics \\
\hline Manuscript ID: & TMPH-2009-0289.R1 \\
\hline Manuscript Type: & Special Issue Paper - In honour of Prof Richard Zare \\
\hline Author: & 06-Jan-2010 \\
\hline Complete List of Authors: & $\begin{array}{l}\text { Bouakline, Foudhil; University of Cambridge } \\
\text { Althorpe, Suart; University of Cambridge } \\
\text { Larregaray, Pascal; Université Bordeaux1/CNRS } \\
\text { Bonnet, Laurent; Université Bordeaux1/CNRS }\end{array}$ \\
\hline Keywords: & $\begin{array}{l}\text { hydrogen-exchange reaction, geometric phase, quasiclassical } \\
\text { trajectories, topology and homotopy, nearside-farside analysis }\end{array}$ \\
\hline $\begin{array}{l}\text { Note: The following files were submitted by the author for peer review, but cannot be converted } \\
\text { to PDF. You must view these files (e.g. movies) online. }\end{array}$ \\
\hline source files.zip
\end{tabular}

\section{s ScholarONE" \\ Manuscript Central}


Molecular Physics

Vol. 00, No. 00, Month 200x, 1-22

\title{
INVITED ARTICLE
}

\section{Strong geometric-phase effects in the hydrogen-exchange reaction at high collision energies: II. Quasiclassical trajectory analysis.}

\author{
Foudhil Bouakline $^{\mathrm{a} *}$, Stuart C. Althorpe ${ }^{\mathrm{a}}$, Pascal Larregaray ${ }^{\mathrm{b}}$, and Laurent Bonnet ${ }^{\mathrm{b}}$ \\ a Department of Chemistry, University of Cambridge, Lensfield Road, Cambridge CB2 \\ 1EW, United Kingdom \\ b Institut des Sciences Moléculaires, Université Bordeaux 1 / CNRS, UMR 5255, 33405 \\ Talence Cedex, France \\ (Received 00 Month 200x; final version received 00 Month 200x)
}

\begin{abstract}
Recent calculations on the hydrogen-exchange reaction [Bouakline et al., J. Chem. Phys. 128, 124322 (2008)], have found strong geometric phase (GP) effects in the state-to-state differential cross sections (DCS), at energies above the energetic minimum of the conical intersection (CI) seam, which cancel out in the integral cross sections (ICS). In this article, we explain the origin of this cancellation and make other predictions about the nature of the reaction mechanisms at these high energies by carrying out quasiclassical trajectory (QCT) calculations. Detailed comparisons are made with the quantum results by splitting the quantum and the QCT cross sections into contributions from reaction paths that wind in different senses around the CI and that scatter the products in the nearside and farside directions. Reaction paths that traverse one transition state (1-TS) scatter their products in just the nearside direction, whereas paths that traverse two transition states (2-TS) scatter in both the nearside and farside directions. However, the nearside 2-TS products scatter into a different region of angular phase-space than the 1-TS products, which explains why the GP effects cancel out in the ICS. Analysis of the QCT results also suggests that two separate reaction mechanisms may be responsible for the 2-TS scattering at high energies.
\end{abstract}

Keywords: hydrogen-exchange reaction; geometric phase; quasiclassical trajectories; topology and homotopy; nearside-farside analysis

\section{INTRODUCTION}

The dynamics of the $\mathrm{H}+\mathrm{H}_{2}$ system and its isotopic variants has been extensively studied both theoretically and experimentally for many decades [1]-[34]. After so many years of study, leading to very good agreement between experimental and quantum mechanical results $[12,25,26]$, and an unprecedented understanding of the reaction at the state-to-state level [28], it is tempting to say that this prototype reaction is fully understood and there is little to learn about it. However, there are still some issues which continue to be discussed such as quantum bottleneck states [20], and also the different reaction mechanisms governing this system [17-19], such as the recently discovered 'tug-of-war' mechanism $[31,32]$ in $\mathrm{H}+\mathrm{D}_{2}$ inelastic scattering.

Another particularly important feature of this system is its well characterized (Jahn-Teller) conical intersection (CI) seam [35]-[37], connecting the electronic

\footnotetext{
* Corresponding author. Email: foudhil.bouakline@googlemail.com

Present address: Institut für Chemie, Universität Potsdam, Karl-Liebknecht-Street, 24-25, D-14476 Potsdam-Golm, Germany

ISSN: 0040-5167 print/ISSN 1754-2278 online

(C) 200x Taylor \& Francis

DOI: $10.1080 / 0040516 Y Y x x x x x x x x$

http://www.informaworld.com
} 
ground state potential energy surface to the first excited state surface by a hyperline passing through the nuclear equilateral triangle geometries. As such, it has been used as a benchmark for understanding non-adiabatic reaction dynamics $[13,30]$ around a CI.

The main role of a conical intersection is to act as a funnel allowing population transfer between the upper and lower adiabatic electronic states via non-radiative transitions [38, 39]. Another accompanying quantum effect is the so-called geometric (or Berry) phase (GP) [40, 41], which occurs even if the nuclear motion is confined to the ground adiabatic electronic state and avoids the neighbourhood of the CI. The GP is the sign-change acquired by the adiabatic electronic wave function when the nuclei complete an odd number of loops around the CI. This affects the nuclear dynamics by introducing a corresponding sign-change in the continuity boundary condition of the nuclear wave function [42]-[45], which cancels out the sign acquired by the adiabatic electronic wave function, required by the singlevaluedness of the total wave function. The effect of the GP on the nuclear wave function of a bound-state system is well understood [45]: It brings changes in the quantum numbers and energy levels with respect to those computed with normal boundary conditions, and this has been observed in many Jahn-Teller molecules [46]-[49]. However, our understanding of such effects in non-bound systems has only become clear recently, owing to detailed reactive scattering calculations and experiments on the hydrogen-exchange reaction [4]-[34], and also on the predissociation of the upper cone-states of the $\mathrm{H}_{3}$ molecular system [50]-[54].

Mead [43] was the first to predict GP effects in the $\mathrm{H}+\mathrm{H}_{2}$ reaction using symmetry arguments. He showed that the GP changes the relative sign of the inelastic and reactive scattering contributions to the fully symmetrized cross sections, whenever the nuclear wave function does not encircle the CI when unsymmetrized. Later work by Kuppermann and co-workers [5],[55]-[58] using multivalued basis functions approach to include the GP, considered more general effects where the unsymmetrized wave function may encircle the CI. Their calculations predicted strong GP effects in the differential cross sections (DCS), but these predictions were neither reproduced by later calculations that omitted the GP $[9,11,24]$, nor by experiments [8, 10]. Detailed experimental results agreed quantitatively well with theoretical predictions ignoring the GP boundary condition. This controversy stimulated further work by Kendrick [11, 59, 60], who performed time-independent quantum calculations and used a different method to include the GP, namely the Mead-Truhlar vector potential approach [42]. He found that GP effects appear in the scattering observables at specific values of the total angular momentum $J$, but cancel on summing over all the partial waves to give the state-to-state integral cross sections (ICS), and also on summing over a limited number of $J(0 \leq J \leq 10)$ to give the low impact parameter DCS.

Subsequent calculations by Juanes-Marcos et al. [24, 61, 62], using a timedependent wave packet method [14] to solve the nuclear Schrödinger equation and the vector potential approach to include the GP, confirmed these results and extended them to high impact parameters to give the fully converged DCS. They found that the DCS do show small GP effects, which cancel on integrating over all the scattering angles to give the ICS. In addition to these calculations, Althorpe and co-workers $[24,61,63]$ were able to explain these findings using topological arguments originated in the late 1960s in Feynman path integral theory in order to explain the analogous Aharonov-Bohm effect [64]-[66]. They demonstrated that the central idea is very simple and can be derived even without path integrals, using symmetry arguments $[24,67]$. They showed that the nuclear wave function can be unwound and separated, without any approximation, into two components, each of 
which contains all the Feynman paths that loop in the relevant sense around the CI. These two components correspond to two different reaction mechanisms in the $\mathrm{H}+\mathrm{H}_{2}$ reaction as shown in figure 1; one passes over one transition state (1-TS) and the other over two transition states (2-TS). The interference between these two components governs the extent to which scattering observables are affected by the GP. In addition, the topological approach of Althorpe [63] predicts that the two reaction paths (1-TS and 2-TS) scatter their products into different regions of space, which causes the cancellation of the GP in the ICS.

In a recent work [30], some of us extended the previous calculations to higher total energies (up to $4.5 \mathrm{eV}$ ), well above the energy minimum of the CI seam occurring at $2.74 \mathrm{eV}$, and took full account of the nonadiabatic coupling to the first excited electronic state. We found little contribution of the excited state to the state-to-state dynamics even at very high energies, and obtained accurate predictions of state-to-state scattering observables using just the ground state surface with inclusion of the geometric phase. The effect of the GP in the state-to-state probabilities, at energies above $3.5 \mathrm{eV}$, were found to be stronger than at low energies, and to survive the coherent sum over the partial waves to produce features in the state-to-state DCS, which could be detected in an experiment with an angular resolution of $20^{\circ}$. However, these effects completely cancel out in the ICS, owing to differences in the slopes of the phases of the 1-TS and 2-TS scattering amplitudes.

The aim of the present article is to investigate the origin of this cancellation of GP effects in the ICS. We do this by carrying out quasiclassical trajectory (QCT) calculations, which allow a more direct and straightforward probing of the reaction mechanisms. We compare the quantum and QCT results in some detail, by decomposing the cross sections into 1-TS and 2-TS contributions, and also, further, into nearside and farside contributions. The methodology used is described in Sec. 2. The results are presented and discussed in Sec. 3. Section 4 concludes the article.

\section{METHODOLOGY}

\subsection{Extraction of the one- and two-transition state mechanisms}

Here we summarize the topological approach [24, 63, 67] which allows us to unwind the nuclear wave function and extract from it the 1-TS and 2-TS Feynman paths. Once the unwound parts are extracted, one can use the 1-TS and 2-TS components to interpret the dynamics and explain the effect of the geometric phase on the different scattering observables. The basic idea of the topological approach is that the nuclear wave function can be split into two parts, $\Psi_{\mathrm{e}}$ and $\Psi_{\mathrm{o}}$. One component $\left(\Psi_{\mathrm{e}}\right)$ is the portion of the wave function which contains all the Feynman paths that complete an even number of clockwise (and an odd number of counterclockwise) loops around the CI; the other part $\left(\Psi_{\mathrm{o}}\right)$ is the sum over all the odd clockwise (and even counterclockwise) looping Feynman paths. The only effect of the GP is to change the relative sign of these two components. In general, it is extremely difficult to extract reaction paths from a nuclear wave function; however, this approach is easy to implement and it only requires [62] the computation of the nuclear wave functions with the GP boundary condition $\left(\Psi_{\mathrm{GP}}\right)$ and with normal boundary conditions ignoring the GP $\left(\Psi_{\mathrm{NGP}}\right)$. Using either symmetry arguments or Feynman paths [63,67], one can show that:

$$
\begin{aligned}
& \Psi_{\mathrm{NGP}}(\alpha)=\frac{1}{\sqrt{2}}\left[\Psi_{\mathrm{e}}(\alpha)+\Psi_{\mathrm{o}}(\alpha)\right] \\
& \Psi_{\mathrm{GP}}(\alpha)=\frac{1}{\sqrt{2}}\left[\Psi_{\mathrm{e}}(\alpha)-\Psi_{\mathrm{o}}(\alpha)\right]
\end{aligned}
$$


where $\alpha$ is any angle such that $\alpha=0 \rightarrow 2 \pi$ describes a closed loop around the CI. It is clear from this equation that GP effects will be noticeable only if the even and odd looping components $\left(\Psi_{\mathrm{e}}\right.$ and $\Psi_{\mathrm{o}}$ ) interfere. From the previous equation, it is trivial to extract the even and odd looping wavefunctions without any approximation:

$$
\begin{aligned}
& \Psi_{\mathrm{e}}(\alpha)=\frac{1}{\sqrt{2}}\left[\Psi_{\mathrm{NGP}}(\alpha)+\Psi_{\mathrm{GP}}(\alpha)\right] \\
& \Psi_{\mathrm{o}}(\alpha)=\frac{1}{\sqrt{2}}\left[\Psi_{\mathrm{NGP}}(\alpha)-\Psi_{\mathrm{GP}}(\alpha)\right]
\end{aligned}
$$

In the $\mathrm{H}+\mathrm{H}_{2}$ reaction, these two components $\left(\Psi_{\mathrm{e}}\right.$ and $\Psi_{\mathrm{o}}$ ) correspond to paths that pass over one transition state (1-TS) and two transition states (2-TS), respectively. So, the terms " $\Psi_{\mathrm{e}}$ paths" and "1-TS paths" (and " $\Psi_{\mathrm{o}}$ path" and "2-TS paths") are equivalent, and will be used interchangeably. Figure 1 illustrates this terminology for the hydrogen-exchange reaction, where the three nuclei are treated as indistinguishable particles. If the reaction enters the encirclement region from the $\mathrm{A}+\mathrm{BC}$ asymptotic arrangement and exits into the $\mathrm{AC}+\mathrm{B}$ arrangement, it is obvious that the 1-TS paths make less than one loop around the CI in the clockwise sense, so they are assigned a winding number $n=0$; the 2-TS paths make less than one revolution, but in the anticlockwise sense, and are assigned a winding number $n=-1$, following the convention of refs.[63, 67]. Therefore, the 1-TS Feynman paths are contained in $\Psi_{\mathrm{e}}$ and the 2-TS paths in $\Psi_{\mathrm{o}}$. The even and odd components of the wave function also contain higher winding numbers, but these are ignored as it is highly unlikely that the nuclei pass over three or more transition states in the $\mathrm{H}+\mathrm{H}_{2}$ reaction.

Since the GP affects the relative phases of $\Psi_{\mathrm{e}}$ and $\Psi_{\mathrm{o}}$, but not these functions individually [62], this topological approach allows us to treat the 1-TS and 2-TS mechanisms as two separate reactions giving rise to their own scattering observables, neither of which is affected by the geometric phase. Consequently, one can make direct comparisons between scattering observables obtained independently from these two components with the corresponding QCT predictions. Separating out the QCT 1-TS and 2-TS mechanisms is easy, since one just counts how many times a given trajectory crosses a transition state (see Fig. 1). For instance, considering trajectories which enter the encirclement region from the $\mathrm{A}+\mathrm{BC}$ asymptotic arrangement (region I) and exit into the $\mathrm{AC}+\mathrm{B}$ arrangement (region II), 1-TS trajectories are the ones ultimately crossing the I/II separation line, whereas 2-TS trajectories ultimately cross the III/II separation line. (Because of the indistinguishability of the three nuclei, an analog analysis is performed for trajectories ending up in the $\mathrm{C}+\mathrm{AB}$ asymptotic arrangement). Almost all trajectories cross a given separation line only once, though complex trajectories involving recrossing exist, but are statistically irrelevant.

We should emphasize that the unwinding technique given by Eq.2 applies to the exact wave function, and the paths we extract from it are different classes of Feynman paths, not classical trajectories. This approach is rigorous, and applies even when the system can access the conical intersection seam (as is done here) and has enough energy to hop between the two surfaces [68].

The GP and NGP wave functions of Eq.2 were calculated using just the ground adiabatic electronic state surface for all total energies. Previous work [13, 30] has shown that the excited state does not contribute appreciably to the dynamics, even at energies above the CI energetic minimum. 


\subsection{Nearside-Farside analysis}

Previous work $[24,30,62]$ at lower energies $(E=2.3 \mathrm{eV})$ found that $\Psi_{\mathrm{e}}$ and $\Psi_{\mathrm{o}}$ scattered into different hemispheres (which was the reason that the GP dephased so efficiently in the ICS): $\Psi_{\mathrm{e}}$ scattered into the "nearside", $\Theta=+\theta$ hemisphere (see Fig. 2), and $\Psi_{\mathrm{o}}$ into the 'farside', $\Theta=-\theta$ hemisphere. This identification was made by noting that the slope of the phase of the $\Psi_{\mathrm{e}}$ scattering amplitude decreased as a function of $\theta$, whereas the slope of the phase of $\Psi_{\mathrm{o}}$ increased with $\theta$. It relied on the fact that each of $\Psi_{\mathrm{e}}$ and $\Psi_{\mathrm{o}}$ was either nearside or farside scattered, and gave a slope that varied more or less linearly with $\theta$ (see Fig. 3). At the higher energies $(E=4.3 \mathrm{eV})$ that we investigate here, this simple analysis is no longer possible, since the phases of the $\Psi_{\mathrm{e}}$ and $\Psi_{\mathrm{o}}$ scattering amplitudes behave in a more complicated way (see Fig. 3 ), suggesting that $\Psi_{\mathrm{e}}$ and $\Psi_{\mathrm{o}}$ probably contain a mixture of nearside and farside components.

In the present work, we carry out a more systematic nearside-farside analysis of $\Psi_{\mathrm{e}}$ and $\Psi_{\mathrm{o}}$ (at $E=4.3 \mathrm{eV}$ ), by making use of semi-classical techniques $[69,70]$ for decomposing each of $\Psi_{\mathrm{e}}$ and $\Psi_{\mathrm{o}}$ scattering amplitudes into nearside- and farsidescattering subamplitudes. It is well known that, in the semiclassical limit $(J \sin \theta \gg$ 1), the state-to-state scattering amplitude becomes

$$
f_{n^{\prime} \leftarrow n}(E, \theta) \simeq f_{n^{\prime} \leftarrow n}^{[+]}(E, \theta)+f_{n^{\prime} \leftarrow n}^{[-]}(E, \theta)
$$

where $f_{n^{\prime} \leftarrow n}^{[+]}$and $f_{n^{\prime} \leftarrow n}^{[-]}$represent the nearside and farside components, respectively. $n$ and $n^{\prime}$ are the composite quantum numbers of the reactant and product diatoms, $E$ is the energy of the system and $\theta$ is the scattering angle. The two components of the scattering amplitude are given by

$$
f_{n^{\prime} \leftarrow n}^{[ \pm]}(E, \theta)=\frac{1}{2 i k_{v j}} \sum_{J}(2 J+1) e_{k^{\prime} k}^{J[ \pm]}(\pi-\theta) S_{n^{\prime} \leftarrow n}(J, E)
$$

and

$$
\left.e_{k^{\prime} k}^{J[ \pm]}(\pi-\theta)=\left[\frac{1}{2 \pi\left(J+\frac{1}{2}\right) \sin \theta}\right]^{1 / 2} \exp \left\{ \pm i\left[\left(J+\frac{1}{2}\right)\right] \theta+\frac{1}{2} \pi\left(k^{\prime}-k\right)-\frac{1}{4} \pi\right]\right\}
$$

where $v$ and $j$ are the vibration and rotation quantum numbers of the reactant diatomic molecule, $k$ and $k^{\prime}$ are the projections of $j$ on the initial and final relative velocity vectors, respectively. $\hbar k_{v j}$ denotes the magnitude of the atom-diatom approach momentum, $J$ the total angular momentum quantum number, and $S_{n^{\prime} \leftarrow n}$ the scattering matrix elements.

The decomposition in Eq.(3) was applied separately to $\Psi_{\mathrm{e}}$ and $\Psi_{\mathrm{o}}$, with the result that the total scattering amplitude $f_{n^{\prime} \leftarrow n}$ was separated into four separate pieces: $f_{n^{\prime} \leftarrow n}^{[e][+]}(1-\mathrm{TS}$, nearside-scattered $), f_{n^{\prime} \leftarrow n}^{[e][-]}(1-\mathrm{TS}$, farside-scattered $), f_{n^{\prime} \leftarrow n}^{[o][+]}(2-$ TS, nearside-scattered) and $f_{n^{\prime} \leftarrow n}^{[0][-]}(2-\mathrm{TS}$, farside-scattered). The same decomposition was also applied to the QCT trajectories. As already mentioned, it is easy to determine whether a trajectory has traversed one or two transition states, and also to determine whether it is nearside or farside-scattered (by testing the sign of the component of the final atom-diatom relative velocity vector parallel to the impact parameter, which is nearside if positive and farside if negative). This analysis was made for all final quantum states $\left(v^{\prime}, j^{\prime}\right)$, making possible detailed comparisons between quantum and QCT results. 


\subsection{Quantum mechanical calculations}

The calculations reported here made use of GP and NGP scattering matrix elements obtained from calculations reported previously [30]. Both sets were calculated using the BKMP2 [71] ground electronic state potential energy surface (PES), with inclusion of the diagonal non-Born-Oppenheimer correction. The calculations were performed using the reactant product decoupling (RPD) method [14], and the geometric phase was incorporated by the Mead-Truhlar vector potential approach [42]. To converge the cross sections for the $\mathrm{H}+\mathrm{H}_{2}(v=0, j=0) \rightarrow \mathrm{H}_{2}\left(v^{\prime}, j^{\prime}\right)+\mathrm{H}$ reaction over total energies ranging from $1.25 \mathrm{eV}$ to $4.5 \mathrm{eV}$, we found it necessary to include all partial waves up to total angular momentum quantum number $J=55$. The hydrogen nuclei are treated as distinguishable particles, ignoring the requirement that the nuclear wave function be antisymmetric under exchange of two ${ }^{1} \mathrm{H}$ nuclei. Thus, our theoretical product distributions and cross sections correspond to the experimentally measurable values only for the odd $-j$ product states.

\subsection{Quasiclassical trajectory calculations}

The quasiclassical trajectory (QCT) calculations were carried out using the same BKMP2 potential energy surface as the quantum calculations. The QCT methodology was adapted from refs.[72, 73]; it uses a standard Monte Carlo sampling of the initial conditions, and the step-adaptive Adams method to integrate the Hamilton equations, where conservation of total energy and total angular momentum were carefully checked. The calculations were performed for two different total energies $(2.3 \mathrm{eV}$ and $4.3 \mathrm{eV})$, where a total of $5 \times 10^{6}$ trajectories were propagated at each energy. The rovibrational quantum numbers $\left(v^{\prime}, j^{\prime}\right)$ of the product outgoing diatomic molecule were assigned, for a given rotational angular momentum, by calculating the exact vibrational action. The Gaussian-weighted (GW) binning method [74]-[76], was employed to extract product distributions, using a full width-at-half-maximum of 0.05 for the Gaussian functions. Unlike the standard histogram binning (SB) method, where the final vibrational action of each trajectory is rounded to its nearest integer, which may lead to inappropriate results, the GW procedure circumvents this problem by weighting each trajectory by a Gaussian-like coefficient, such that the closer the final vibrational action is to an integer, the larger the coefficient. It has been proven [74]-[76] that the GW procedure gives more realistic results than the SB technique, especially when the total energy disposal in the products is too low for the classical and quantum densities of vibrational states to be equal. However, test calculations on our system showed negligible differences between the GW and SB techniques for only high final vibrational states and for a total energy of $2.3 \mathrm{eV}$, and almost the same results for a total energy of $4.3 \mathrm{eV}$.

The separation into 1-TS and 2-TS trajectories was done by converting to the hyperspherical coordinate system of Kuppermann [77], and counting how many times each trajectory crossed a transition state. The resulting 1-TS and 2-TS scattering cross sections were then further decomposed into nearside and farside-scattered components, to permit direct comparison with the quantum results (obtained as in Sec. 2.2). 


\section{RESULTS AND DISCUSSION}

\subsection{Overview and summary of previous results}

Quantum results were obtained from the S-matrix elements of ref. [30] at energies 2.3 and $4.3 \mathrm{eV}$ as described in Sec. 2. The overall effect of the GP in the $2-4.5 \mathrm{eV}$ total energy range is summarised in Fig. 4. At $2 \mathrm{eV}$, the contribution from 2-TS scattering is very small indeed, although it does make small differences to some of the DCS by interfering with the 1-TS scattering, as described in refs. [30, 62]. By $3.5 \mathrm{eV}$, however, the 2-TS scattering is now of roughly equal importance to the 1-TS scattering (the overall ratio of 1 -TS:2-TS at $4.3 \mathrm{eV}$ is $3: 1$ ). This results in GP effects in the DCS as described in ref. [30] and illustrated in Fig. 5 (for one particular final state). At $2.3 \mathrm{eV}$, the 2-TS component is very small indeed and produces no noticeable contribution to the DCS (although for some other final states it does make small differences $[30,62])$. At $4.3 \mathrm{eV}$, the interference between the 1-TS and 2-TS contributions produces strong GP effects in the DCS.

\subsection{Comparison with QCT results and nearside-farside decomposition}

Figures 6 and 7 show comparisons between the quantum and QCT integral cross sections (rotational product distributions for each $v^{\prime}$ ) at total energies of 2.3 and $4.3 \mathrm{eV}$. At $2.3 \mathrm{eV}$, the QCT and quantum results are in good overall agreement, and reproduce results reported earlier in ref. [62]. At $4.3 \mathrm{eV}$, the QCT and quantum results are also in good agreement, although the QCT overestimate the bimodal shape of the product distributions at $v^{\prime}=0,1$. In the quantum calculations, this shape is barely evident, as two shallow maxima (see Fig. 7), but in the classical calculations the maxima are pronounced.

An explanation for the above discrepancy is given when the separate 1-TS and 2-TS distributions are compared (at $4.3 \mathrm{eV}$ ) in Fig. 8. As mentioned above, the separation of these two classes of mechanism is easy to make, and involves no approximation. At $2.3 \mathrm{eV}$, the results (not shown) are in reasonable agreement, reproducing those of ref. [62]. At this energy, only about $2 \times 10^{-4} \%$ of the trajectories pass over two transition states, so the 2-TS contribution to the ICS is very small. At $4.3 \mathrm{eV}$, however, the 1-TS and 2-TS contributions are of roughly the same magnitude. Figure 8 shows that the 1-TS and 2-TS QCT results reproduce well the corresponding quantum results. The bimodal shape is seen to be a direct consequence of the fact that the 1-TS rotational distributions are hotter than the 2-TS distributions at low $v^{\prime}$, resulting in two peaks, which are more pronounced in the QCT case. However, the agreement between the quantum and classical 1-TS and 2-TS distributions is on the whole very good.

This agreement is seen to continue when each of the 1-TS and 2-TS cross sections is decomposed further, into nearside and farside contributions. In the 1-TS cross sections, the farside contribution to both the quantum and classical results is negligible (and not shown here). In the 2-TS cross sections, the nearside and farside contributions are of equal magnitude. Figure 9 shows that the quantum and QCT predictions of these quantities agree on the whole very well. As $v^{\prime}$ increases, the importance of the farside contribution increases; at $v^{\prime}=0$ the nearside contribution is dominant, at $v^{\prime}=3$ the farside contribution is roughly twice that of the nearside. 


\subsection{Origin of the GP cancellation in the ICS}

By this stage in the analysis, we have split the state-to-state ICS into 4 pieces indicating the sense in which the trajectory winds about the CI and the hemisphere into which it scatters, and have found excellent agreement between the quantum and $\mathrm{QCT}$ results. This gives us confidence that the QCT results provide a faithful description of the dynamics, subject to the caveat that the QCT rotational distributions will tend to be hotter than the quantum distributions. We can therefore use the QCT results to explain why the difference between the 1-TS and 2-TS scattering is such that, at $4.3 \mathrm{eV}$, the GP effects that are present in the state-to-state (quantum) DCS cancel out in the corresponding ICS.

Let us first remind ourselves why a similar cancellation occurs at $2.3 \mathrm{eV}$. Figure 10 shows the angular distribution of trajectories as a function of total angular momentum (i.e. initial impact parameter). It is clear that the majority of the 1-TS trajectories scatter into the nearside $(\Theta=+\theta)$ hemisphere, whereas the majority of the 2-TS scatter into the farside $(\Theta=-\theta)$ hemisphere. As a result, the 1-TS-2-TS overlap integral is highly oscillatory as a function of $\theta$, which causes the GP effects (in the quantum results) to average out to a very small value on integrating over $\theta$ to give the ICS.

Figure 11 shows the angular distribution at $4.3 \mathrm{eV}$. This is consistent with Fig. 9 in showing that the 2-TS trajectories now scatter into both the nearside and farside hemispheres. However, it is clear from this plot that the nearside 2-TS trajectories scatter into a different region of angular phase-space to the 1-TS trajectories. The 2-TS nearside trajectories scatter from relatively low impact parameters $(J=0 \rightarrow$ 23) into the forward direction $\theta=0 \rightarrow 60^{\circ}$, whereas the 1-TS trajectories scatter from high impact parameters $(J=30 \rightarrow 47)$ into this range of angles. As a result, the 1-TS-2-TS overlap integral is still highly oscillatory, and integrating over $\theta$ to give the (quantum) ICS causes an efficient averaging out to zero of the GP effects in the DCS.

A full characterisation of the mechanisms responsible for the 1-TS and 2-TS scattering at $4.3 \mathrm{eV}$ is difficult (not least because it does not make sense to talk about neatly defined 'reaction mechanisms' at such high energies), and will not be attempted here. Nevertheless, we can pick out some general features from Fig. 11 that give clues into how the dynamics at $4.3 \mathrm{eV}$ differ from the much better understood dynamics at $2.3 \mathrm{eV}$. First, the 1-TS trajectories no longer scatter into the recoil direction $\theta \sim 180^{\circ}$, even from low impact parameters. This is not surprising, since the simple recoil mechanism that produces scattering into these angles at lower energies is unlikely to operate at energies as high as $4.3 \mathrm{eV}$. Second, the low impact $(J<25)$ 2-TS trajectories behave very differently from the high impact $(J>25)$ trajectories. The former scatter in roughly equal proportions into the nearside and farside hemispheres, with low $v^{\prime}(<2)$ favouring the nearside hemisphere, and high $v^{\prime}$ the farside (see also Fig. 9). The high impact 2-TS trajectories, by contrast, scatter entirely into the farside direction. This suggests that the latter are closer to the direct 'insertion' mechanism followed by the lower-energy 2-TS trajectories, in which the attacking $\mathrm{H}$ atom first weakens, and then passes through the reactant $\mathrm{H}-\mathrm{H}$ bond.

\section{CONCLUSIONS}

We have carried out detailed comparisons of quantum and QCT reactive scattering cross sections for the hydrogen-exchange reaction at high energies $(4.3 \mathrm{eV})$, and found excellent agreement between the two sets of results. This agreement continues 
to hold when the cross sections are decomposed into contributions from 1-TS and 2-TS reaction paths (which procedure is rigorous and based on topology), and when the latter are further decomposed into nearside and farside-scattering contributions (which decomposition is approximate in the quantum calculations, and makes use of a semiclassical approximation to the scattering wave function).

As a result, we can trust the QCT results to give a faithful description of the quantum scattering at these high energies. In particular, inspection of the QCT angular distributions answers the question raised by previous work [30], as to why GP effects in the high energy $(>3.5 \mathrm{eV})$ DCS cancel out in the ICS. Unlike at lower energies $(<3.5 \mathrm{eV})$, the 1 -TS and 2 -TS paths both scatter products into the nearside hermisphere; however, the nearside 2-TS paths scatter into a different region of angular phase-space than the 1-TS paths, and thus result in a 1-TS-2-TS interference term which is oscillatory as a function of $\theta$. As a result the GP effects cancel out in the ICS.

Further work will be needed to characterise the nature of the reaction mechanisms at these higher energies. We have shown here that the QCT calculations are sufficient to describe these mechanisms, including those properties that are responsible for the cancellation of GP effects in the ICS.

The results of this paper confirm those of ref. [30] in predicting that the best way to detect GP effects experimentally in the hydrogen-exchange reaction is to measure the the state-to-state DCS at high energies $(>3.5 \mathrm{eV})$. Measurements of the ICS at these energies will not contain noticeable GP effects, although measurement of the $v^{\prime}=0$ rotational distribution would allow an experiment to distinguish between 1-TS and 2-TS products.

\section{Acknowledgements}

F.B. and S.C.A. acknowledge the support of this work by the UK Engineering and Physical Sciences Research Council (EPSRC). P.L. acknowledges fruitful discussions with Eckart Wrede.

\section{References}

[1]G. C. Schatz and A. Kuppermann, J. Chem. Phys. 65, 4642 and 4668 (1976).

[2]D. G. Truhlar and R. E. Wyatt, Ann. Rev. Phys. Chem. 27, 1 (1976).

[3] G. C. Schatz, Ann. Rev. Phys. Chem. 39, 317 (1988).

[4]W. H. Miller, Annu. Rev. Phys. Chem. 41, 245 (1990).

[5]B. Lepetit and A. Kuppermann, Chem. Phys. Lett. 166, 581 (1990).

[6]W. H. Miller and J. Z. H. Zhang, J. Phys. Chem. 95, 12 (1991).

[7] M. J. D'Mello, D. E. Manolopoulos, and R. E. Wyatt, Science 263, 102 (1994).

[8]E. Wrede and L. Schnieder, J. Chem. Phys. 107, 786 (1997).

[9] M. P. de Miranda, D. C. Clary, J. F. Castillo, and D. E. Manolopoulos, J. Chem. Phys. 108, 3142 (1998).

[10]E. Wrede, L. Schnieder, K. H. Welge, F. J. Aoiz, L. Bañares, J. F. Castillo, B. Martínez-Haya, and V. J. Herrero, J. Chem. Phys. 110, 9971 (1999).

[11]B. K. Kendrick, J. Chem. Phys. 112, 5679 (2000).

[12] F. Fernández-Alonso, B. D. Bean, J. D. Ayers, A. E. Pomerantz, R. N. Zare, L. Bañares, and F. J. Aoiz, Angew. Chem., Int. Ed. 39, 2748 (2000).

[13] S. Mahapatra, H. Köppel, and L. S. Cederbaum, J. Phys. Chem. A 105, 2321 (2001).

[14]S. C. Althorpe, J. Chem. Phys. 114, 1601 (2001).

[15]F. Fernández-Alonso, B. D. Bean, R. N. Zare, F. J. Aoiz, L. Bañares, and J. F. Castillo, J. Chem. Phys. 115, 4534 (2001).

[16]F. Fernández-Alonso and R. N. Zare, Annu. Rev. Phys. Chem. 53, 67 (2002).

[17]S. C. Althorpe, F. Fernández-Alonso, B. D. Bean, J. D. Ayers, A. E. Pomerantz, R. N. Zare, and E. Wrede, Nature (London) 416, 67 (2002).

[18]S. A. Harich, D. Dai, C. C. Wang, X. Yang, S. D. Chao, and R. T. Skodje, Nature (London) 419, 281 (2002).

[19] D. Dai, C. C. Wang, S. A. Harich, X. Wang, X. Yang, S. D. Chao, and R. T. Skodje, Science 300, 1730 (2003). 
[20]R. T. Skodje and X. M. Yang, Int. Rev. Phys. Chem. 23, 253 (2004).

[21]F. Ausfelder, A. E. Pomerantz, R. N. Zare, S. C. Althorpe, F. J. Aoiz, L. Bañares, and J. F. Castillo, J. Chem. Phys. 120, 3255 (2004).

[22] A. E. Pomerantz, F. Ausfelder, R. N. Zare, S. C. Althorpe, F. J. Aoiz, L. Bañares, and J. F. Castillo, J. Chem. Phys. 120, 3244 (2004).

[23] K. Koszinowski, N. T. Goldberg, A. E. Pomerantz, R. N. Zare, J. C. Juanes-Marcos, and S. C. Althorpe, J. Chem. Phys. 123, 054306 (2005).

[24]J. C. Juanes-Marcos, S. C. Althorpe, and E. Wrede, Science 309, 1227 (2005).

[25]F. J. Aoiz, L. Bañares, and V. J. Herrero, Int. Rev. Phys. Chem. 24, 119 (2005).

[26]L. Bañares, F. J. Aoiz, and V. J. Herrero, Phys. Scr. 73, C6 (2006).

[27] M. Hankel, S. C. Smith, R. J. Allan, S. K. Gray, and G. G. Balint-Kurti, J. Chem. Phys. 125, 164303 (2006).

[28]K. Koszinowski, N. T. Goldberg, J. Zhang, R. N. Zare, F. Bouakline, and S. C. Althorpe, J. Chem. Phys. 127, 124315 (2007).

[29] B. Jayachander Rao, R. Padmanaban, and S. Mahapatra, Chem. Phys. 333, 135 (2007).

[30]F. Bouakline, S. C. Althorpe, and D. Peláez Ruiz, J. Chem. Phys. 128, 124322 (2008).

[31]S. J. Greaves, E. Wrede, N. T. Goldberg, J. Zhang, D. J. Miller, and R. N. Zare, Nature 454, 91 (2008).

[32] N. T. Goldberg, J. Zhang, K. Koszinowski, F. Bouakline, S. C. Althorpe, and R. N. Zare, PNAS 105, 18194 (2008).

[33]S. J. Greaves, D. Murdock, E. Wrede, and S. C. Althorpe, J. Chem. Phys. 128, 164306 (2008).

[34] P. D. D. Monks, J. N. L. Connor, and F. Bouakline, J. Phys. Chem. A 113, 4746 (2009).

[35] A. J. C. Varandas, F. B. Brown, C. A. Mead, D. G. Truhlar, and N. C. Blais, J. Chem. Phys. 86, 6258 (1987).

[36] R. Abrol, A. Shaw, A. Kuppermann, and D. R. Yarkony, J. Chem. Phys. 115, 4640 (2002).

[37] C. R. Evenhuis, X. Lin, D. H. Zhang, D. Yarkony, and M. A. Collins, J. Chem. Phys. 123, 134110 (2005).

[38] Conical Intersections: Electronic Structure, Dynamics and Spectroscopy, edited by W. Domcke, D. R. Yarkony, and H. Köppel (World Scientific, River Edge, NJ, 2003).

[39] G. A. Worth and L. S. Cederbaum, Annu. Rev. Phys. Chem. 55, 127 (2004).

[40] G. Herzberg and H. C. Longuet-Higgins, Discuss. Faraday Soc. 35, 77 (1963).

[41] M. V. Berry, Proc. R. Soc. London, Ser. A 392, 45 (1984).

[42] C. A. Mead and D. G. Truhlar, J. Chem. Phys. 70, 2284 (1979).

[43] C. A. Mead, J. Chem. Phys. 72, 3839 (1980).

44] C. A. Mead, Rev. Mod. Phys. 64, 51 (1992).

[45] M. S. Child, Adv. Chem. Phys. 124, 1 (2002).

[46]B. K. Kendrick, Phys. Rev. Lett. 79, 2431 (1997).

[47] H. von Busch, V. Dev, H.-A. Eckel, S. Kasahara, J. Wang, W. Demtröder, P. Sebald, and W. Meyer, Phys. Rev. Lett. 81, 4584 (1998).

[48] B. E. Applegate, T. A. Barckholtz, and T. A. Miller, Chem. Soc. Rev. 32, 38 (2003).

[49]D. Babikov, B. K. Kendrick, P. Zhang, and K. Morokuma, J. Chem. Phys. 122, 044315 (2005).

[50]B. Lepetit, Z. Peng, and A. Kuppermann, Chem. Phys. Lett. 166, 572 (1990).

[51]S. Mahapatra and H. Köppel, J. Chem. Phys. 109, 1721 (1998).

[52] A. J. C. Varandas and L. P. Viegas, Chem. Phys. Lett. 367, 625 (2003).

[53]B. Lepetit, R. Abrol, and A. Kuppermann, Phys. Rev. A 76, 040702(R) (2007).

[54]F. Bouakline, B. Lepetit, S. C. Althorpe, and A. Kuppermann, in The Jahn-Teller Effect: Fundamentals and Applications for Physics and Chemistry, ed. by H. Köppel, D. R. Yarkony, and H. Barentzen (Springer-Verlag, Springer Series in Chemical Physics, Vol. 97, 2010), pp 201-238.

[55] Y.-S. M. Wu, A. Kuppermann, and B. Lepetit, Chem. Phys. Lett. 186, 319 (1991).

[56] A. Kuppermann and Y.-S. M. Wu, Chem. Phys. Lett. 205, 577 (1993).

[57] A. Kuppermann and Y.-S. M. Wu, Chem. Phys. Lett. 241, 229 (1995).

[58] A. Kuppermann and Y.-S. M. Wu, Chem. Phys. Lett. 349, 537 (2001).

[59] B. K. Kendrick, J. Phys. Chem. A 107, 6739 (2003).

[60]B. K. Kendrick, J. Chem. Phys. 118, 10502 (2003).

[61] J. C. Juanes-Marcos and S. C. Althorpe, J. Chem. Phys. 122, 204324 (2005).

[62] J. C. Juanes-Marcos, S. C. Althorpe, and E. Wrede, J. Chem. Phys. 126, 044317 (2007).

[63] S. C. Althorpe, J. Chem. Phys. 124, 084105 (2006).

[64]L. S. Schulman, Phys. Rev. 176, 1558 (1968).

[65]L. S. Schulman, J. Math. Phys. 12, 304 (1971).

[66] M. G. G. Laidlaw and C. M. De Witt, Phys. Rev. D 3, 1375 (1971)

[67] S. C. Althorpe, J. C. Juanes-Marcos, and E. Wrede, Adv. Chem. Phys. 138, 1 (2008)

[68] S. C. Althorpe, T. Stecher, and F. Bouakline, J. Chem. Phys. 129, 214117 (2008).

[69] M. S. Child, Molecular Collision Theory (Dover, New York, 1996).

[70] A. J. Dobbyn, P. McCabe, J. N. L. Connor, and J. F. Castillo, Phys. Chem. Chem. Phys. 1, 1115 (1999).

[71]A. I. Boothroyd, W. J. Keogh, P. G. Martin, and M. R. Peterson, J. Chem. Phys. 104, 7139 (1996).

[72]D. G. Truhlar and J. T. Muckerman, in Atom-Molecule Collision Theory, edited by R. B. Bernstein (Plenum, New York, 1979).

[73] P. Halvick and J.-C. Rayez, Chem. Phys. 131, 375 (1989).

[74] L. Bonnet and J.-C. Rayez, Chem. Phys. Lett. 277, 183 (1997).

[75] L. Bonnet and J.-C. Rayez, Chem. Phys. Lett. 397, 106 (2004).

[76] L. Bonnet, J. Chem. Phys. 128, 044109 (2008).

[77] A. Kuppermann, Chem. Phys. Lett. 32, 374 (1975). 
Figure captions

Figure 1: Schematic representation of the 1-TS (solid) and 2-TS (dashed) reaction paths in the reaction $\mathrm{H}_{\mathrm{A}}+\mathrm{H}_{\mathrm{B}} \mathrm{H}_{\mathrm{C}} \rightarrow \mathrm{H}_{\mathrm{A}} \mathrm{H}_{\mathrm{C}}+\mathrm{H}_{\mathrm{B}}$. The $\mathrm{H}_{3}$ potential energy surface is represented using the hyperspherical coordinate system of Kuppermann (Ref. [77]), in which the equilateral-triangle geometry of the CI is in the center $(\times)$ and the linear transition states $(\ddagger)$ are on the perimeter of the circle. The angle $\alpha$ is the internal angular coordinate which describes motion around the CI. The dashed (red) lines separate the three different atom-diatom channels.

Figure 2: Diagram illustrating "nearside" scattering (positive deflection angles $\Theta)$ and "farside" scattering (negative deflection angles). The arrow (chains) represents the initial approach direction of the reagents in the center-of-mass frame; the gray rectangle represents the spread of impact parameters in the initial plane wave.

Figure 3: (Color online). Phases of the $(v=0, j=0) \rightarrow\left(v^{\prime}=2, j^{\prime}=1, \Omega^{\prime}=0\right)$ 1-TS (solid line) and 2-TS (dashed line) scattering amplitudes.

Figure 4: (Color online). $(v=0, j=0) \rightarrow\left(v^{\prime}, j^{\prime}\right)$ quantum state-to-state ICS (solid line), 1-TS (dashed line) and 2-TS (dashed-dotted line) contributions.

Figure 5: (Color online). (a) State-to-state DCS for $(v=0, j=0) \rightarrow\left(v^{\prime}=\right.$ $\left.2, j^{\prime}=1\right)$ computed using the lower adiabatic PES including the diagonal nonadiabatic correction without (solid line) and with (dashed line) the GP. (b) State-tostate DCS for $(v=0, j=0) \rightarrow\left(v^{\prime}=2, j^{\prime}=1\right)$ obtained by extracting the 1-TS (solid line) and 2-TS (dashed line) contributions to the GP and non-GP scattering amplitudes. For clarity, the 2-TS DCS are shown multiplied by a factor of 200 for a total energy of $2.3 \mathrm{eV}$.

Figure 6: (Color online). Quantum (closed square solid line) and QCT (closed circle solid line) ICS at a total energy of $2.3 \mathrm{eV}$, for $(v=0, j=0) \rightarrow\left(v^{\prime}, j^{\prime}\right)$.

Figure 7: (Color online). Quantum (closed square solid line) and QCT (closed circle solid line) ICS at a total energy of $4.3 \mathrm{eV}$, for $(v=0, j=0) \rightarrow\left(v^{\prime}, j^{\prime}\right)$.

Figure 8: (Color online). Quantum (closed square solid line) and QCT (closed circle solid line) 1-TS and 2-TS state-to-state ICS at a total energy of $4.3 \mathrm{eV}$, for $(v=0, j=0) \rightarrow\left(v^{\prime}, j^{\prime}\right)$.

Figure 9: (Color online). Quantum mechanical and QCT "nearside" and "farside" components of the 2-TS state-to-state ICS at $4.3 \mathrm{eV}$ total energy.

Figure 10: (Color online). Density plot of the correlation between the deflection angle and the total angular momentum $J$ for 1-TS (open squares) and 2-TS (open circles) QCT trajectories at $2.3 \mathrm{eV}$ total energy. Note that all the 2-TS and only 10000 1-TS trajectories are plotted.

Figure 11: (Color online). Density plot of the correlation between the deflection angle and the total angular momentum $J$ for 1-TS (open squares) and 2-TS (open circles) QCT trajectories at $4.3 \mathrm{eV}$ total energy. Note that, for clarity, only 10000 trajectories are plotted for each type. 


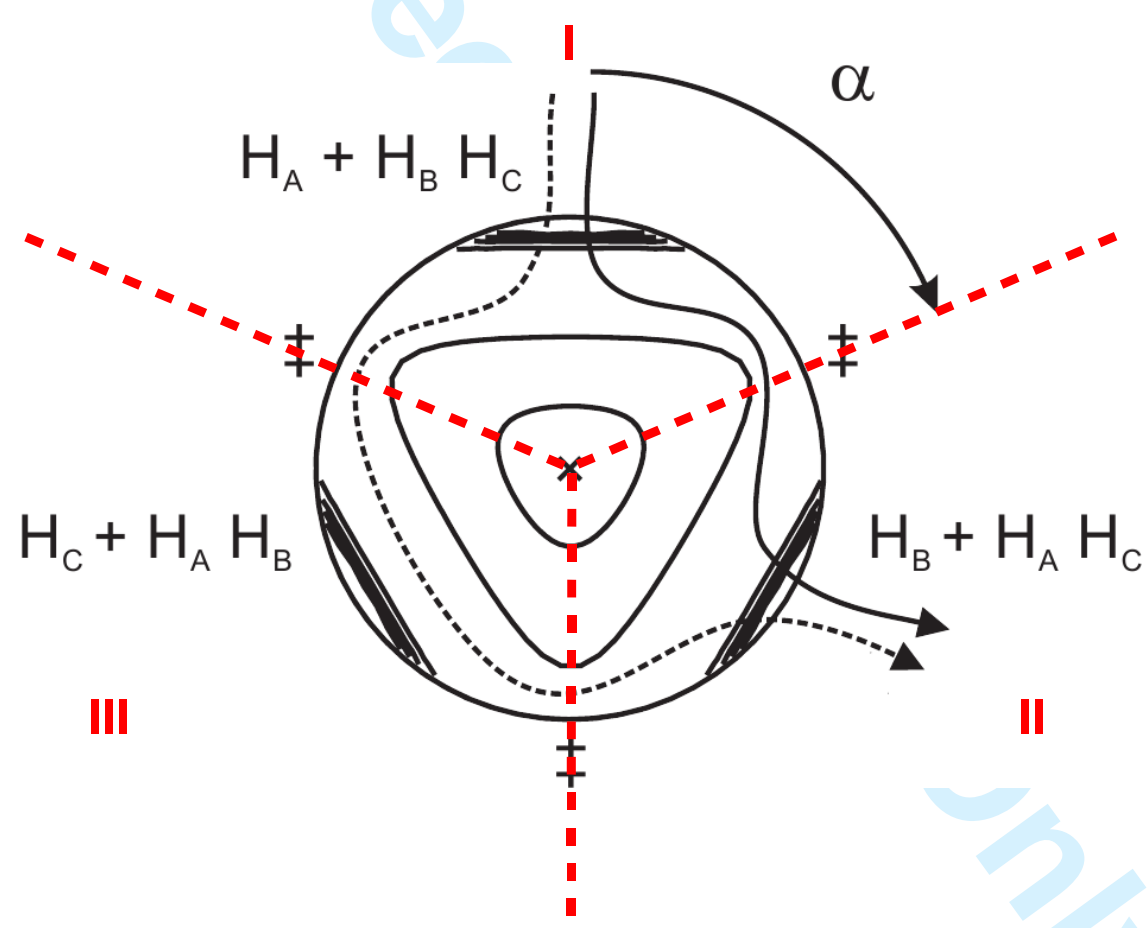

Figure 1. 


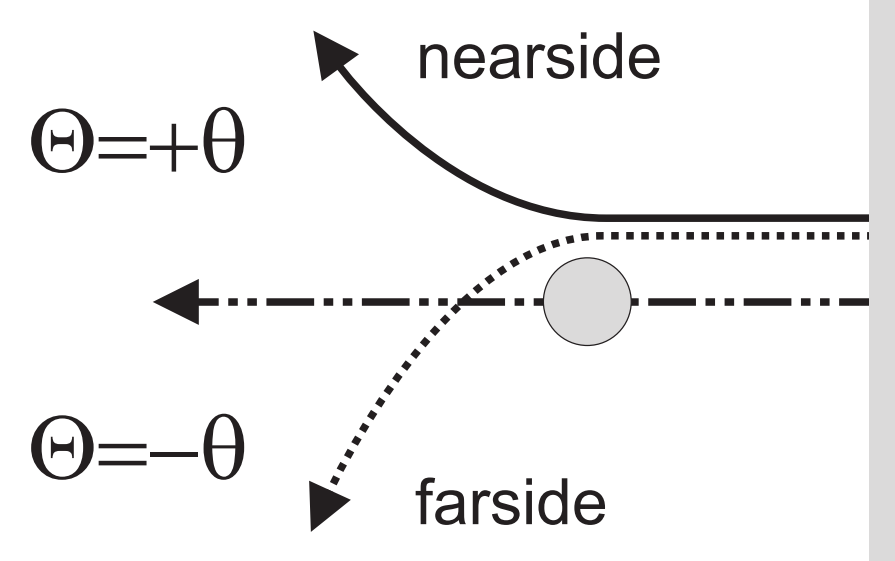

Figure 2. 


\section{Molecular Physics}

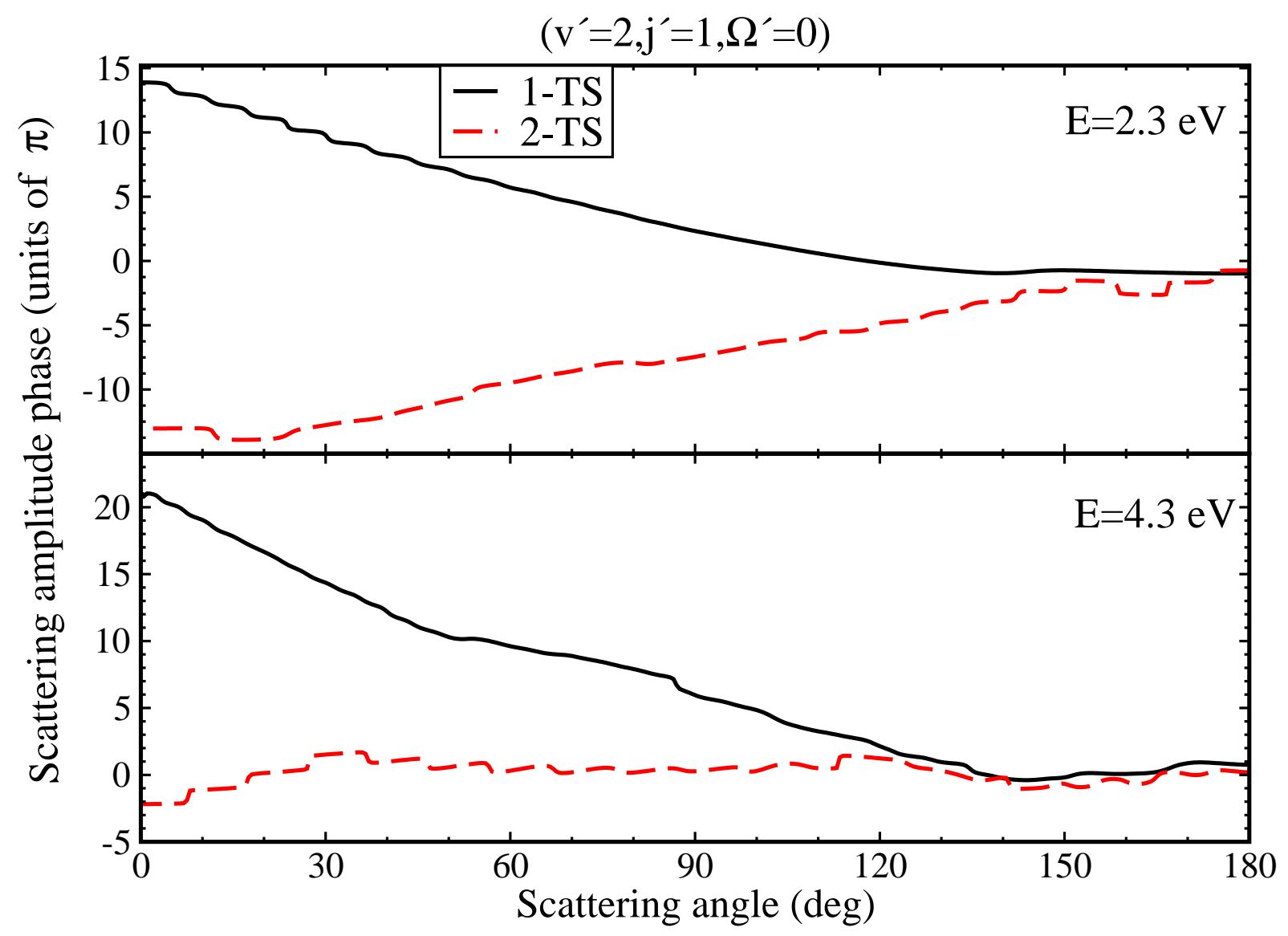

Figure 3. 


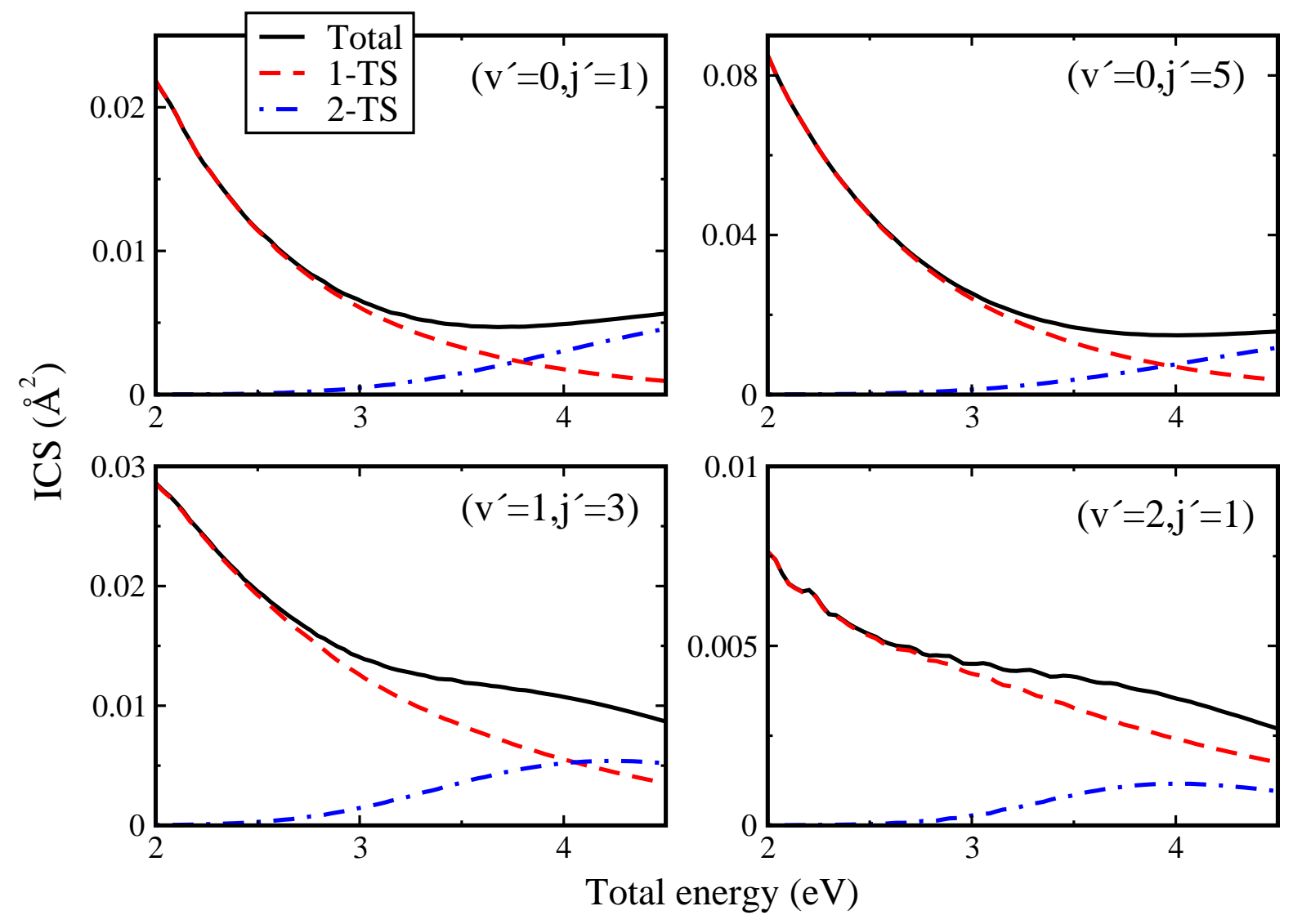

Figure 4. 


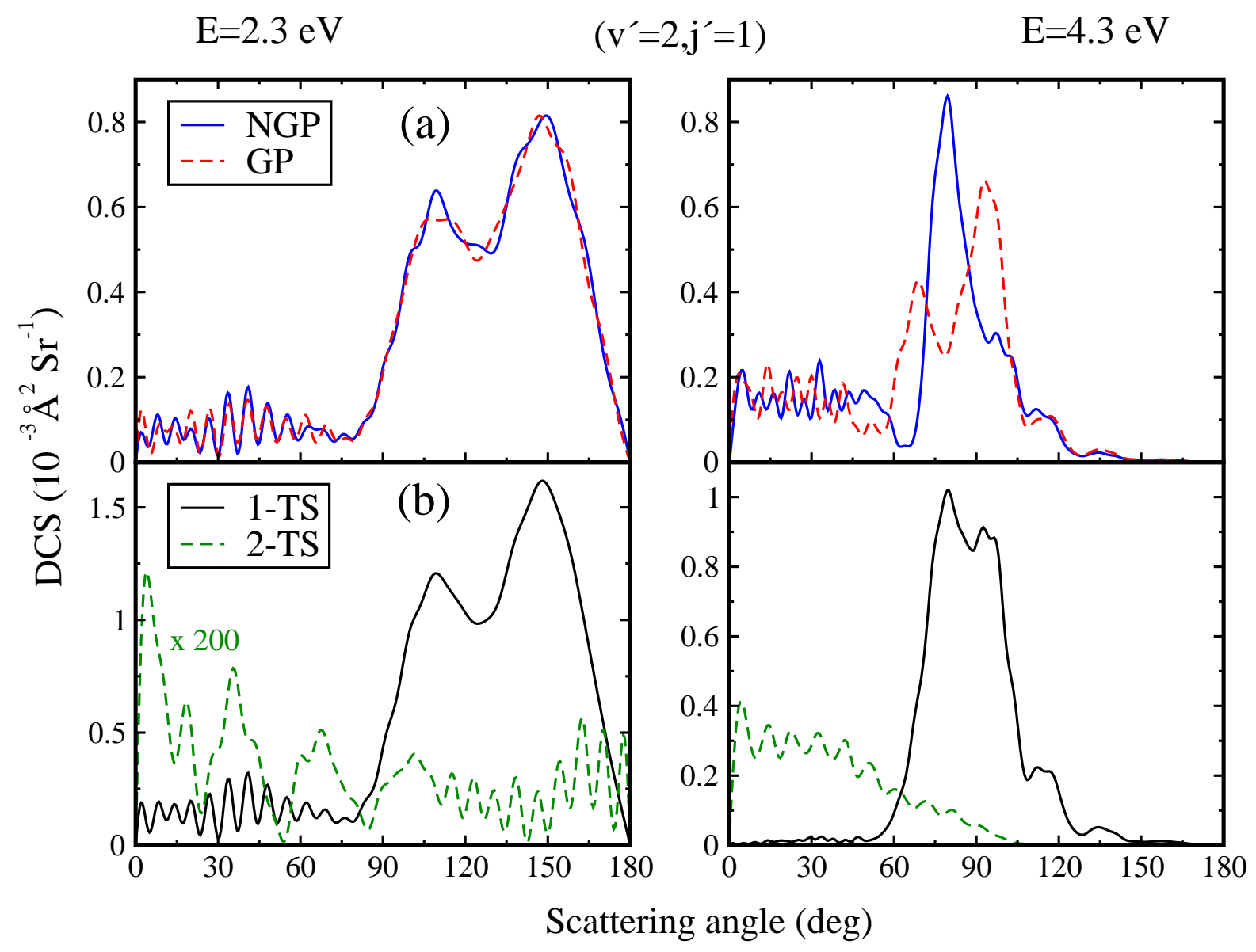

Figure 5. 


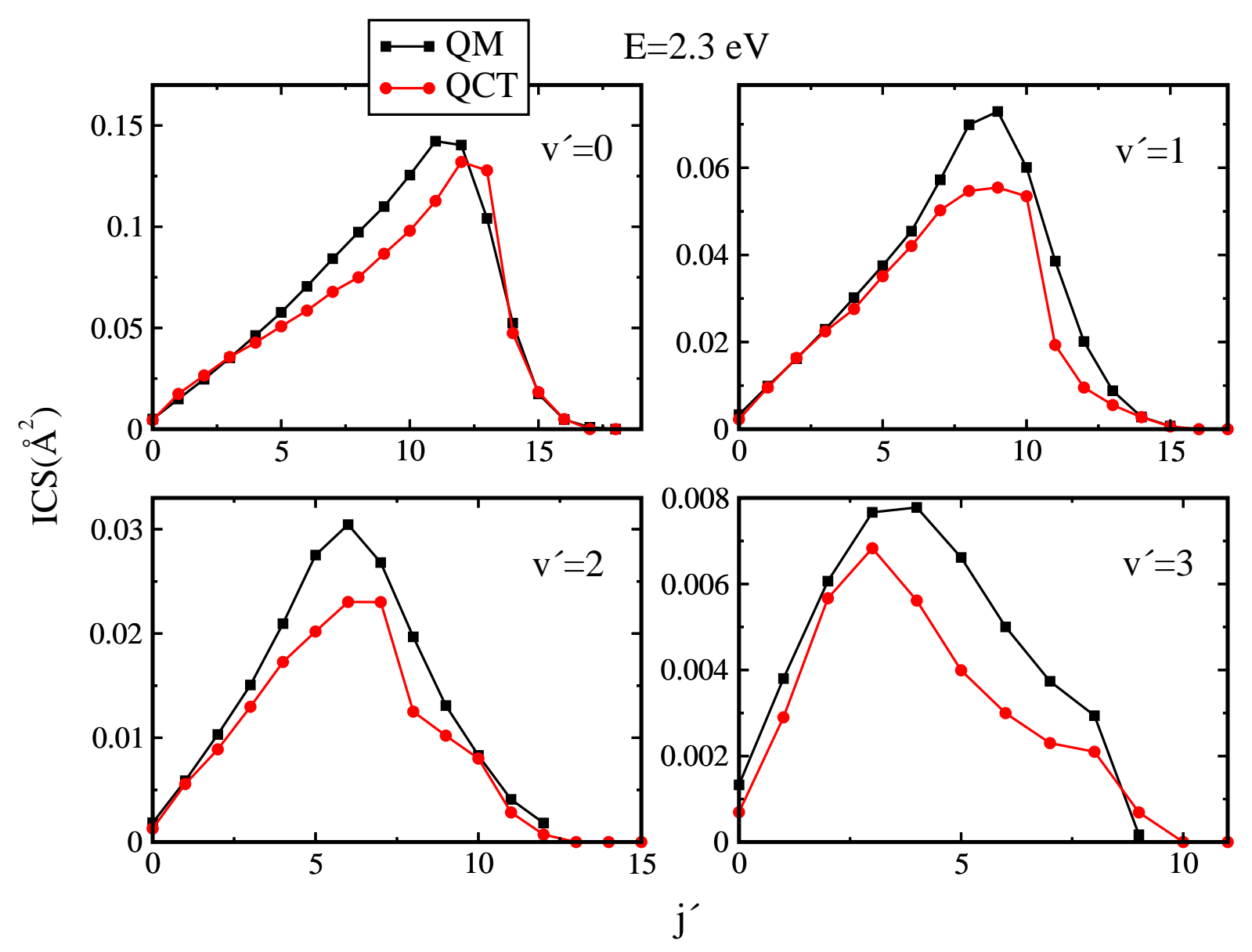

Figure 6. 


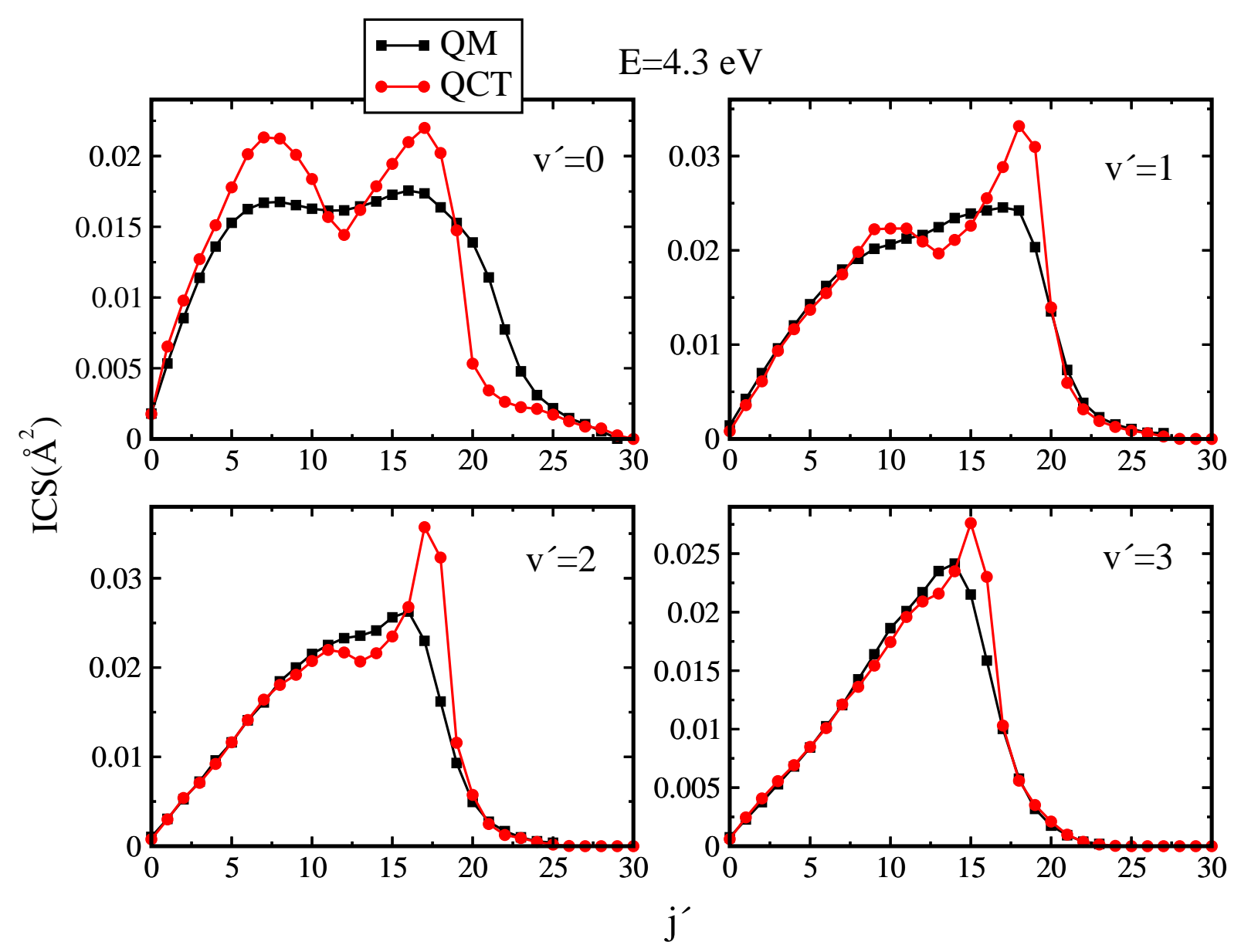

Figure 7. 


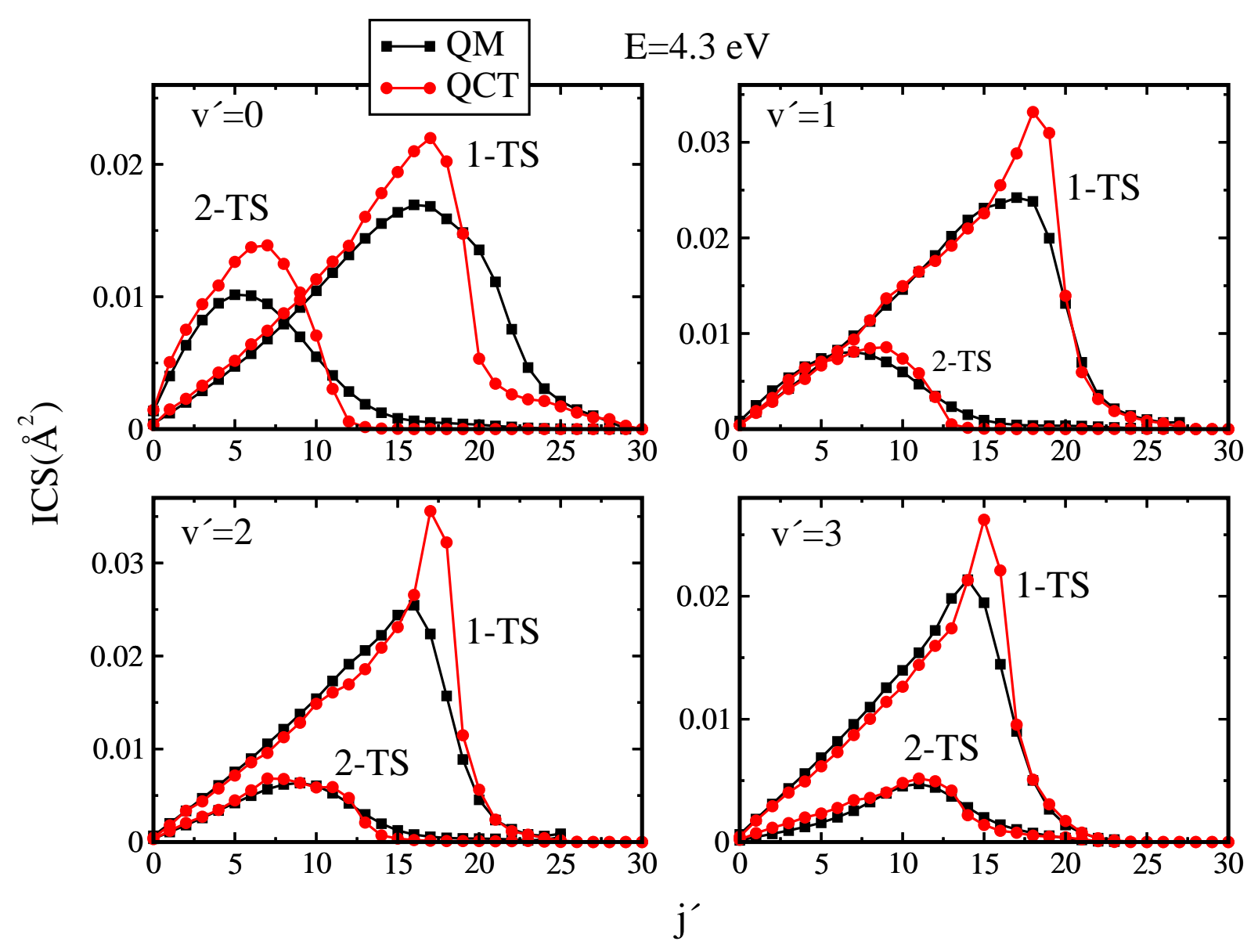

Figure 8. 


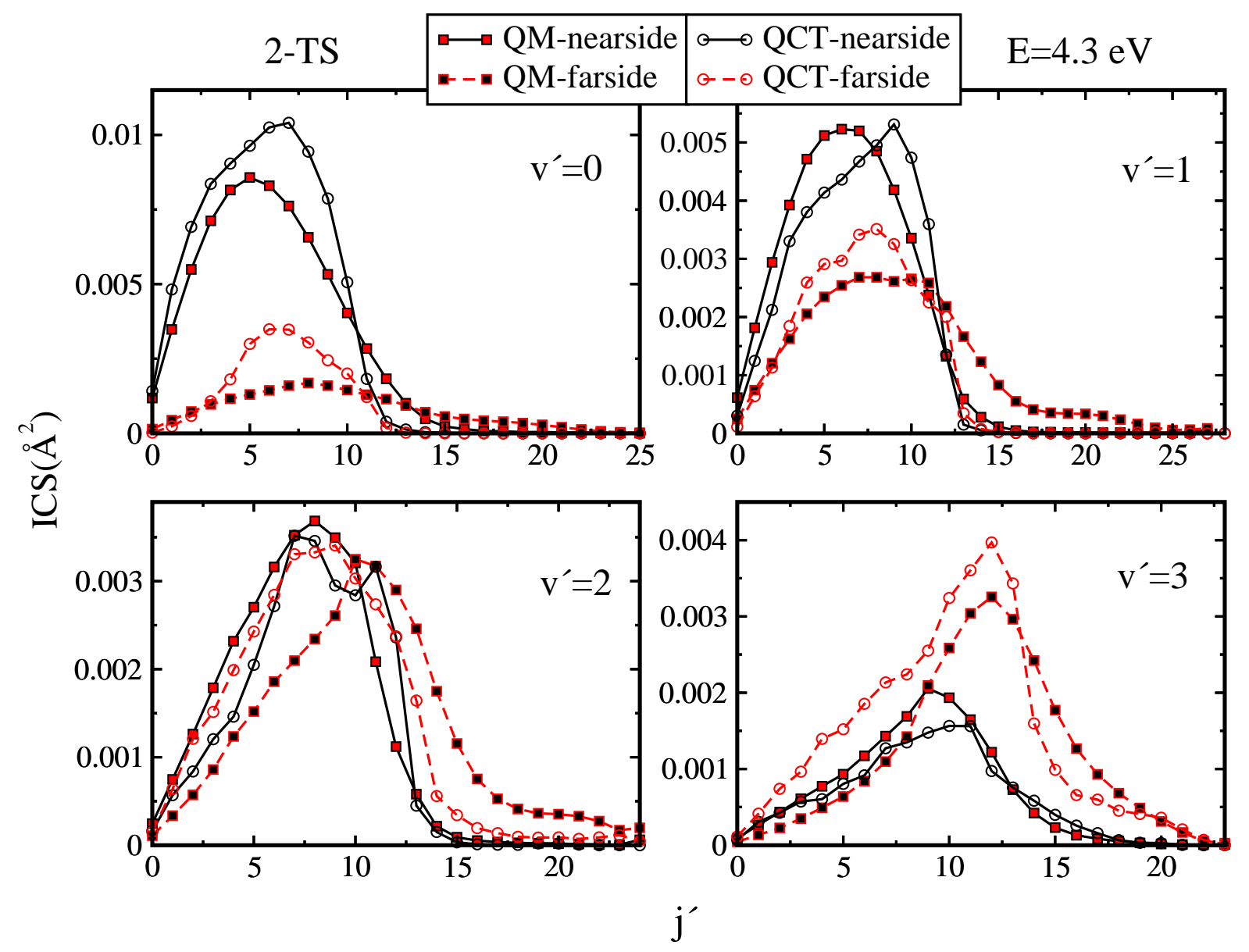

Figure 9. 


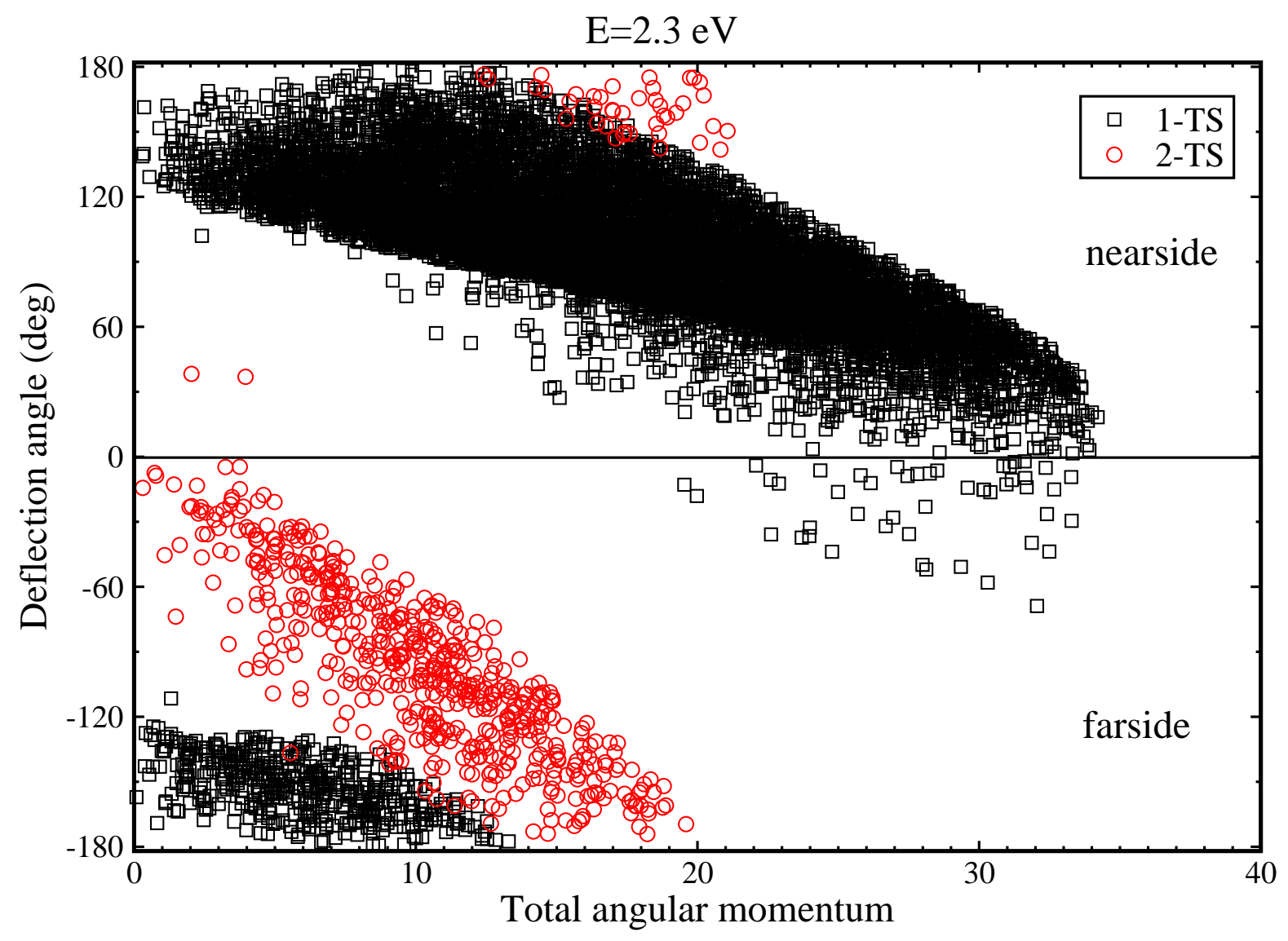

Figure 10. 


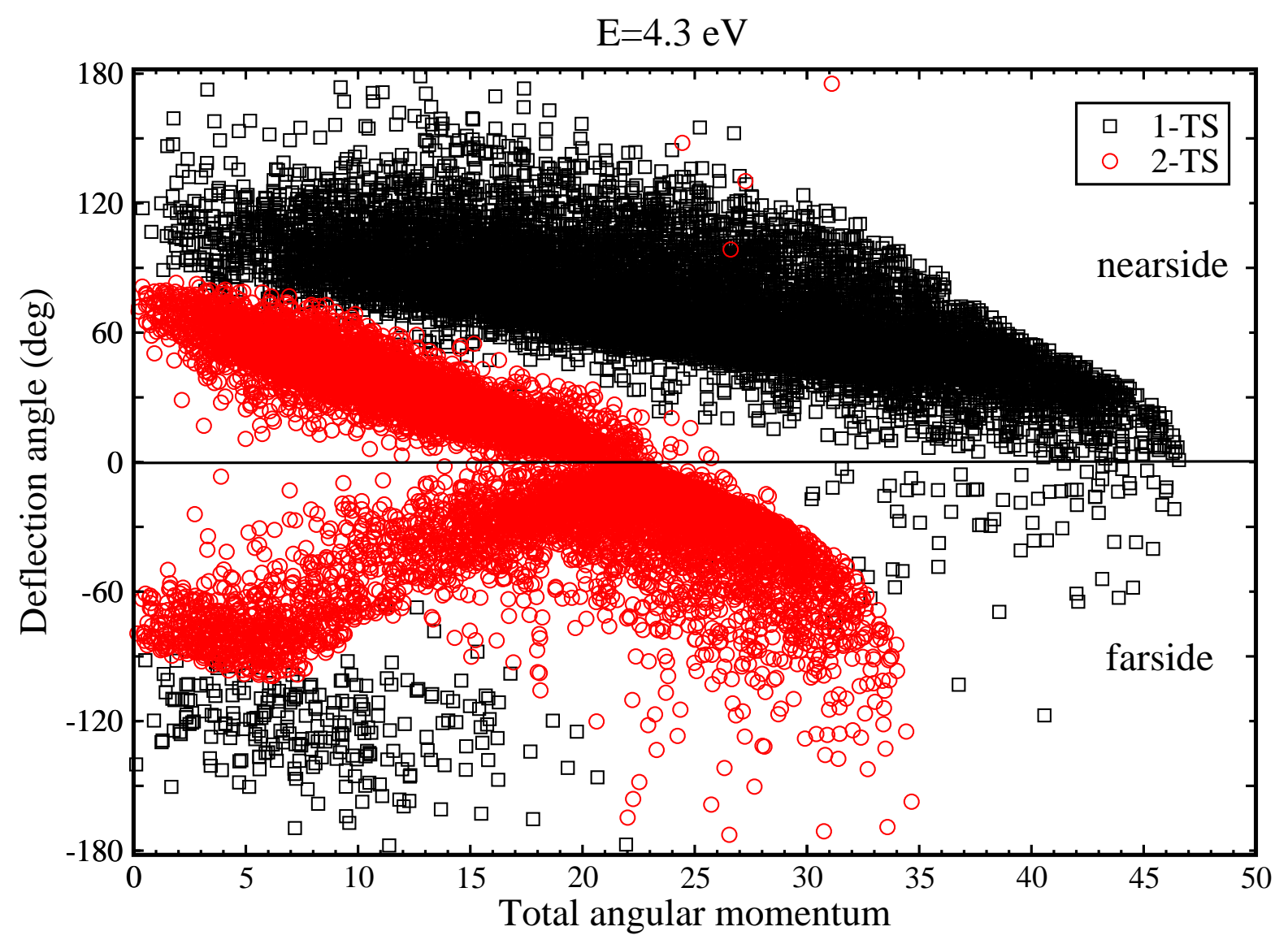

Figure 11. 